\title{
LIPOFUSCIN OF THE RETINAL PIGMENT EPITHELIUM: A REVIEW
}

\author{
CHRIS J. KENNEDY, PIROSKA E. RAKOCZY and IAN J. CONSTABLE \\ Nedlands, Australia
}

\begin{abstract}
SUMMARY
Accumulation of lipofuscin is one of the most characteristic features of ageing observed in retinal pigment epithelial (RPE) cells. The lipofuscin found in RPE cells differs from that of other body tissues due to the fact that it is mainly derived from the chemically modified residues of incompletely digested photoreceptor outer segments. It is a heterogeneous material composed of a mixture of lipids, proteins, and different fluorescent compounds, the main fluorophore of which has recently been identified as a derivative of vitamin A. Research interest has variously focussed on the roles of age, light damage, free radicals, antioxidants, visual pigments, retinal locus, lysosomal enzymes, and pigmentation on lipofuscin formation, as well as the effects of lipofuscin on RPE cell function and causation of retinal disease. This article reviews the recent advances in knowledge of the composition, origin, and possible deleterious effects of RPE cell lipofuscin.
\end{abstract}

Generally regarded as a sign of cellular ageing or senescence, lipofuscin is not a distinct chemical compound but the generic name given to an autofluorescent, membrane-bound intracellular material that is distributed widely amongst the post-mitotic cells of different organs of the body. With advancing age, there is a marked increase in the lipofuscin granule content of human retinal pigment epithelial (RPE) cells and there now exists substantial evidence that the bulk of this lipofuscin represents the chemically modified residues of incompletely digested photoreceptor outer segments. ${ }^{1-4}$ The origin of the lipofuscin in the retinal pigment epithelium therefore differs significantly from that of the lipofuscin in other tissues. Apart

From: Molecular Biology Unit, Lions Eye Institute, Centre for Ophthalmology and Visual Science, University of Western Australia, Nedlands, Western Australia.

Correspondence to: Dr Chris J. Kennedy, 71 Davies Road, Claremont, Western Australia, 6010. from its unique derivation, the lipofuscin of the retinal pigment epithelium differs from that of the brain, heart, testes, and other organs in both electron density and uniformity of granule size. ${ }^{5}$ The composition, origin, and possible deleterious effects of RPE cell lipofuscin have been topics of considerable scientific interest and controversy in recent years and this article reviews current knowledge in these areas.

\section{MORPHOLOGY AND TOPOGRAPHY}

RPE cell lipofuscin is contained within granules of relatively uniform size and homogeneous electron density. 5 The granules are typically more numerous in the basal half of the RPE cell around the nucleus. As noted below, with increasing age there is a tendency for lipofuscin granules to fuse with melanosomes to form complex granules. These have a high electron density core of melanin surrounded by a lipofuscin coating of lower electron density (Fig. 1). The topographic distribution of lipofuscin across the fundus has been found to parallel the density of rod outer segments (ROS). Macular RPE cells contain greater amounts of lipofuscin than non-macular RPE cells, with the exception of a focal reduction in lipofuscin concentration at the fovea. ${ }^{2,6,7}$ The concentration of complex granules within the retinal pigment epithelium is similarly highest at the macula and progressively decreases through the equator to the periphery. ${ }^{1}$

\section{FLUORESCENT CHARACTERISTICS}

Lipofuscin is composed of a mixture of different fluorophores, reflecting the heterogeneous nature of this cellular pigment. Ten different fluorescent fractions have been identified within chloroform extractions of RPE cell lipofuscin. ${ }^{8}$ All these fluorophores have common absorption peaks in the 


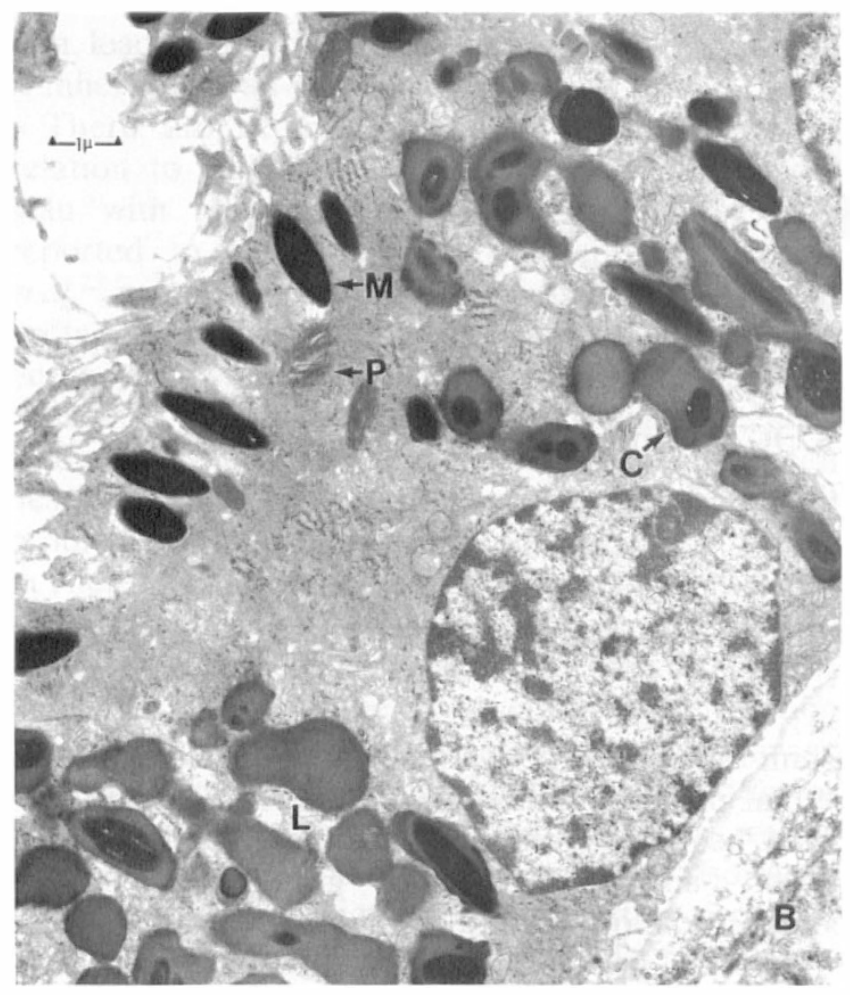

Fig. 1. Transmission electron micrograph of the macular retinal pigment epithelium of a 51-year-old Caucasian male. Phagosome $(P)$ containing rod outer segment material, lipofuscin granules $(L)$, melanosome $(M)$, complex granule (C) containing lipofuscin with a central melanin core and Bruch's membrane $(B)$ can be seen. $(\times 7255)$.

ultraviolet range of the spectrum around $280-330 \mathrm{~nm}$ and fall into four broad categories based on their fluorescent excitation and emission characteristics (Table I). Earlier reports of RPE lipofuscin containing significant blue-emitting fluorophores were found to be incorrect. With the use of carefully calibrated fluorescence instrumentation, both in situ lipofuscin granules and the chloroform extracts of lipofuscin granules or lipofuscin-laden tissues have a peak in emission spectra around 570-605 nm (golden-yellow) when excited at $366 \mathrm{~nm} .{ }^{9,10}$

\section{THE CHEMICAL COMPOSITION OF RPE} CELL LIPOFUSCIN

Although the histochemical characteristics and chloroform:methanol solubility of RPE cell lipofuscin had previously indicated that it was mainly

Table I. Fluorophores present in the chloroform extracts of RPE cell lipofuscin ${ }^{8}$

\begin{tabular}{|c|c|c|}
\hline Fluorophores & $\begin{array}{l}\text { Excitation } \\
\text { peaks }(\mathrm{nm})\end{array}$ & $\begin{array}{l}\text { Emission } \\
\text { peaks }(\mathrm{nm})\end{array}$ \\
\hline Two green-emitting fractions & 330 & 520 \\
\hline $\begin{array}{l}\text { Three yellow/green-emitting } \\
\text { fractions }\end{array}$ & 280,330 & 568 \\
\hline $\begin{array}{l}\text { One golden-yellow-emitting } \\
\text { fraction }\end{array}$ & 280,330 & 585 \\
\hline $\begin{array}{l}\text { Four orange/red-emitting } \\
\text { fractions }\end{array}$ & $285,335,420$ & $605,633,670$ \\
\hline
\end{tabular}

composed of lipid, only recently has more detailed knowledge of the chemical composition of lipofuscin been acquired. ${ }^{11}$ Whilst the proteinaceous content is still unknown, some of the lipid components of RPE lipofuscin have now been analysed and found to differ significantly from the lipid composition of the adjacent retina and the ROS that are phagocytosed by the RPE cells. ${ }^{12}$ The major fatty acids of lipofuscin have been shown to be palmitic, arachidonic, and oleic acids. Also, the major orangeemitting fluorophore of RPE lipofuscin has recently been identified, using fast-atom bombardment tandem mass spectrometry, as $N$-retinylidene- $N$-retinylethanolamine, an amphoteric quaternary amine that arises as a Schiff base reaction product of ethanolamine and retinaldehyde. ${ }^{13}$ Ethanolamine is a component of the ubiquitous membrane lipid phosphatidylethanolamine and retinaldehyde is, of course, the oxidised form of vitamin A which is combined with opsin to form the photopigment rhodopsin of the ROS. Previous animal experiments in which vitamin A intake was manipulated had strongly indicated a role for vitamin A in lipofuscin formation. Dietary vitamin A deficiency had been shown virtually to eliminate the orange-emitting fluorophore of lipofuscin ${ }^{14}$ and to reduce the number of lipofuscin granules in RPE cells of rats. ${ }^{15}$ It appears that vitamin A deficiency primarily reduces lipofuscin accumulation by diminishing photoreceptor outer segment turnover and hence ROS phagocytosis by RPE cells. ${ }^{16}$ The remaining unidentified fluorophores contained in RPE lipofuscin appear to be structurally related to each other and may also consist of modified retinoids. ${ }^{11}$

\section{THE ORIGIN OF RPE CELL LIPOFUSCIN}

Each RPE cell apposes around 30-50 photoreceptor outer segments in vivo and $10-15 \%$ of each outer segment is phagocytosed and replaced each day. ${ }^{17,18}$ In order for RPE cells to eliminate successfully these vast quantities of discs synthesised by the photoreceptor inner segments, the RPE cells must phagocytose and digest the ROS with great efficiency. Phagocytosis and digestion of ROS occurs in several discrete stages (Fig. 2). ${ }^{19}$ The first stage, ROS binding, involves recognition and attachment of ROS to the RPE cell. This is followed by the activation of intracellular microtubules and microfilaments which endocytose cell surface-bound ROS to form phagosomes. ${ }^{20}$ Lysosomes then fuse with these membrane-bound particles to form phagolysosomes or secondary lysosomes. Digestion of the ROS by lytic enzymes then proceeds to yield compounds that are small enough to diffuse out of the phagolysosome. ${ }^{2}$ RPE cell lysosomes contain many lytic enzymes and these include cathepsin D, cathepsin B, $\alpha$-mannosidase, $N$-acetyl- $\beta$-D-glucos- 


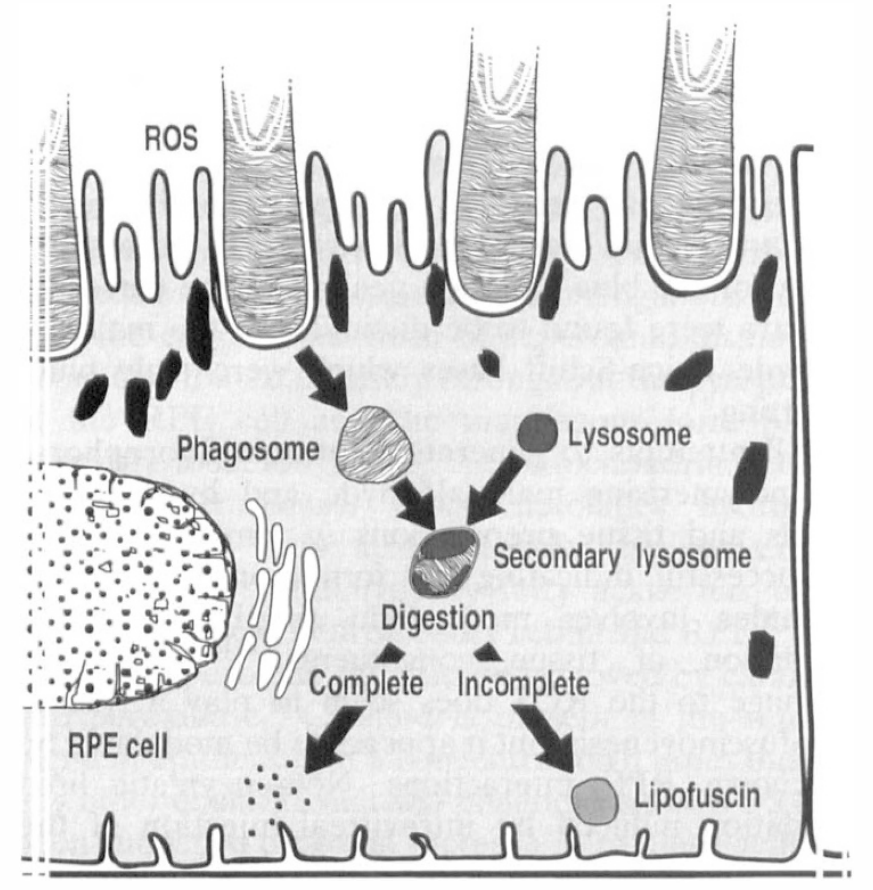

Fig. 2. Retinal pigment epithelial (RPE) cell processing of rod outer segments (ROS).

aminidase, acid lipase, acid phosphatase, $\alpha$-fucosidase, $\alpha$-galactosidase, $\alpha$-glucosidase, $\beta$-galactosidase, $\beta$-glucosidase, $\beta$-glucuronidase, and phospholipases $\mathrm{A}_{1}$ and $\mathrm{A}_{2} \cdot{ }^{21-24}$ The recently identified RPE protease cathepsin $\mathrm{S}$ has not been localised to the lysosome, but appears to be important in ROS digestion. ${ }^{25}$ This complex array of lysosomal enzyme within the RPE cell is believed to be specialised for the purpose of ROS digestion. RPE lysosomal enzymes are, for example, around 7 times more efficient than the lysosomal enzymes of the liver in degrading ROS. ${ }^{22}$ Nevertheless, since all current evidence indicates that RPE lipofuscin represents an altered form of the indigestible residues of lysosomal enzymatic digestion of phagocytosed ROS, ${ }^{7,26-28}$ it appears that the lysosomal enzyme system of the RPE cell is not absolutely effective in fulfilling its task of degrading all the ROS.

It is possible that lipofuscin is caused to accumulate by alterations in either the ROS or the RPE cell
(Table II). It has been theorised that if ROS are oxidatively damaged prior to phagocytosis, the RPE lysosomal system may fail to digest them adequately because the aberrant molecular species no longer match active sites on the degradative enzymes. ${ }^{7,29,30}$ Such undigested oxidised residues within the RPE cells may then be further altered to form lipofuscin. The amount of oxidative damage to the ROS and hence the rate of RPE cell lipofuscin accumulation could potentially be influenced by factors such as the levels of retinal oxygenation and light exposure (potentiating free radical formation) and the antioxidant systems within the retina. ${ }^{29}$ Alternatively, it is possible that primary failure of the action of the RPE lysosomal enzymes could lead to an accumulation of ROS-derived residues that then undergo further modification to form lipofuscin. ${ }^{4}$ The evidence to support each of these theories is outlined below.

\section{EVIDENCE THAT LIPOFUSCIN IS DERIVED FROM PHOTORECEPTOR OUTER SEGMENTS}

Lipofuscin-like autofluorescent pigment has been found to accumulate within cultured human RPE cells as a consequence of ROS phagocytosis in vitro. ${ }^{3,31}$ In vivo, elimination of photoreceptor outer segments from the retina by selective lightinduced destruction has been shown to reduce lipofuscin accumulation in RPE cells. ${ }^{32}$ In the Royal College of Surgeons rat, the RPE cells have a genetic defect in ROS phagocytosis and contain lower levels of lipofuscin than congenic age-matched controls. ${ }^{33}$

Despite all the evidence that the precursors of RPE cell lipofuscin are supplied by ROS, it should be stated that no experiment has yet succeeded in tracing chemical groups all the way from ROS to lipofuscin granules. Immunohistochemical studies targeting opsin have failed to confirm that any of this ROS-derived protein ends up in lipofuscin granules following the phagocytosis and lysosomal enzyme digestion of ROS by RPE cells. ${ }^{27}$ The most probable reason for this is that the lysosomal enzyme

Table II. Factors that are known or suspected to influence the rate of lipofuscin formation in the retinal pigment epithelium

\begin{tabular}{ll}
\hline Factors retarding lipofuscin formation & Factors promoting lipofuscin formation \\
\hline Elimination of rod outer segments & Rod outer segment oxidative damage \\
Vitamin A deficiency & Light exposure (photochemical effects) \\
Ocular melanin & High oxygen content of retina \\
Retinal antioxidants & Chemical pro-oxidants (e.g. iron) \\
Vitamin C & Antioxidant deficiency \\
Vitamin E & Vitamin E deficiency \\
Superoxide dismutase & Lysosomal enzyme defects \\
Glutathione peroxidase & Protease inhibitors \\
Peroxidase & Increasing age \\
Catalase & \\
Reduction in dietary calories or food intake & \\
\hline
\end{tabular}


digestion process rapidly alters or destroys the superficial hydrophilic antigen-binding sites on the opsin molecule for which the antibodies are specific.

\section{PHOTORECEPTOR OUTER SEGMENT OXIDATIVE DAMAGE}

Oxidative damage to the retinal tissues can result from light and the natural by-products of oxidative metabolism. ${ }^{29,34}$ Light energy transmitted through the ocular media can cause damage to the retina through both thermal effects and photochemical reactions. It is the photochemical effects, which are greater with shorter-wavelength light, that result in oxidative damage and these damaging effects are greatly potentiated by the involvement of oxygen. Transfer of an electron to oxygen results in the formation of the highly reactive superoxide free radical. Subsequent free radicals formed can include singlet oxygen and the hydroxyl radical, and these can all initiate photodynamic chain reactions. ${ }^{29}$ Since the outer segment discs and plasma membranes of the photoreceptors are rich in polyunsaturated fatty acids and the ease with which fatty acids undergo auto-oxidation is proportional to their degree of unsaturation, ROS are particularly vulnerable to auto-oxidation. ${ }^{35,36}$ Also, oxygen is concentrated in the fatty acid layers of the ROS discs where it is highly soluble. ${ }^{29}$ Peroxidation of unsaturated lipid bonds, which can be initiated by several types of free radical, results in the formation of alkyl lipids. As these alkyl lipids are also free radicals, these species can convert neighbouring unsaturated fatty acids into alkyl lipids and propagate a chain reaction which will spread throughout the lipid bilayer of individual ROS disc membranes. ${ }^{5}$ ROS membranes containing such oxidatively altered lipids continue to be ingested by the RPE cell as the phagocytic process is apparently unimpaired by these chemical alterations.

It was previously believed by many that lipofuscin was simply a lysosomal residue representing the combination of products of photoreceptor outer segment auto-oxidation. These products were thought to be comprised of malonaldehyde, lipid peroxides and other related chemical species. The prevalent lipid peroxidation theory of lipofuscinogenesis was based on the papers of Chio et al. in $1969 .{ }^{37,38}$ Malonaldehyde is the principal and most characteristic breakdown product of lipid peroxidation. It is highly reactive and forms irreversible crosslinks through the formation of Schiff bases with the amino groups of a wide variety of macromolecules such as DNA, phospholipids and proteins in a nonspecific manner. ${ }^{5}$ These early experiments compared the fluorescence spectra of lipofuscin with those of lipid peroxidation products created by the incubation of subcellular organelles with oxygen.
Several imine-Schiff bases were created from malonaldehyde and amino acids as a result of lipid peroxidation, and the investigators found them to have similar blue fluorescent emission spectra to those they had recorded for lipofuscin. However, instrument bias was later discovered to be responsible for the blue emission peaks and the corrected spectra were found to be dissimilar to the malonaldehyde imine-Schiff bases which were truly blueemitting. ${ }^{11}$

All attempts to generate lipofuscin fluorophores by polymerising malonaldehyde and by oxidising lipids and tissue preparations in vitro have been unsuccessful, indicating that formation of lipofuscin granules involves more than simply the direct oxidation of tissue constituents.,9,39 Oxidative damage to the ROS does seem to play a role in lipofuscinogenesis, but it appears to be modulated by unknown RPE interactions. Non-enzymatic lipid oxidation induced by intravitreal injection of the catalyst iron sulphate in rats in vivo has been shown to result in the appearance of golden-yellow-emitting lipofuscin-like autofluorescent pigment within the RPE cells only after the phagocytosis of oxidised ROS. The oxidised ROS alone did not show these characteristics prior to phagocytosis, thus supporting the concept that the insoluble lipofuscin fluorophores form as a result of a further modification of ingested oxidised ROS material. ${ }^{40}$

\section{EFFECT OF LIGHT EXPOSURE AND MELANIN CONTENT OF FUNDUS}

The lipofuscin content of RPE cells has been found to be significantly greater in whites than in blacks. The inverse relationship between lipofuscin content of RPE cells and choroidal melanin suggests that melanin may have a protective role in preventing lipofuscin formation. The fundi of Caucasians reflect around $5 \%$ of incident white light versus only $1 \%$ in blacks. $^{7}$ The increase in reflected photons in Caucasians may lead to an increase in light-induced free radical formation, increased ROS oxidative damage and hence greater rate of lipofuscin accumulation. Animal experiments have certainly correlated higher levels of light exposure with increased rates of lipofuscin accumulation. ${ }^{41}$ Melanin granules are thought to play a protective role by absorbing radiation and possibly by directly scavenging free radicals and quenching excited molecular states produced by the photochemical and photodynamic effects of radiation. ${ }^{1,29}$

\section{THE ROLE OF ANTIOXIDANTS}

Several antioxidant systems exist in the outer retina and retinal pigment epithelium to deal with the free radicals that confront these cells. They include the cellular enzymes superoxide dismutase, glutathione 
peroxidase, peroxidase, and catalase, and the vitamins $\mathrm{E}$ ( $\alpha$-tocopherol) and C (ascorbate).

Superoxide dismutase, which catalyses the conversion of superoxide to hydrogen peroxide, has been found to be concentrated in ROS. ${ }^{29}$ RPE cells also contain two distinct isoenzymes of superoxide dismutase: a copper-zinc and a manganous form, with the copper-zinc form of superoxide dismutase being distributed diffusely throughout the cytoplasm of the RPE cell and the manganous form being primarily localised within the mitochondria. ${ }^{42}$ Glutathione peroxidase, which detoxifies hydrogen peroxide and fatty acid peroxides by converting them to water and hydroxy fatty acids, has been identified in both neurosensory retina and RPE. ${ }^{5,29,35}$ Hydrogen peroxide can also be removed by catalase and peroxidase. ${ }^{5}$ Catalase is present in the retinal pigment epithelium at a concentration 6 times that in any other ocular tissue, and phagocytosis of ROS has been shown to cause an increase in catalase activity in cultured human RPE cells. ${ }^{43}$

Acting as a free radical scavenger, vitamin E can absorb unpaired free electrons to terminate autooxidation chain reactions. ${ }^{5,35}$ Vitamin $\mathrm{E}$ is known to be concentrated in the lipid phase of ROS and in vitro experiments have shown that vitamin $\mathrm{E}$ can reduce lipid hydroperoxide formation in ROS exposed to light. ${ }^{29}$ In rats deficient in vitamin $\mathrm{E}$, but receiving an adequate vitamin $\mathrm{A}$ intake, there is a marked increase in lipofuscin-like granules within the RPE cells. ${ }^{15}$ Diets deficient in vitamin $\mathrm{E}$ have been shown to result in an enhanced rate of lipofuscin-like granule accumulation in RPE cells of monkeys and rats, especially when the diet is high in polyunsaturated fatty acids. ${ }^{5,15,44}$ Also, it has been reported that the severity of oxygen-induced retinopathy in rats may be diminished by supplementing the animals' diets with vitamin $E .^{45}$ Although vitamin E deficiency causes an accumulation of autofluorescent lipofuscin-like granules in RPE which seem to be derived from ROS, ${ }^{32}$ there is some evidence to suggest that these pigments are in fact dissimilar to lipofuscin due to their different organ distribution, extractability and chromatographic characteristics. $^{11,46}$

Like vitamin $\mathrm{E}$, vitamin $\mathrm{C}$ can also terminate oxidative chain reactions and quench singlet oxygen. ${ }^{29}$ It has been demonstrated that exposure of baboon and guinea pig neural retina to light results in an increase in oxidised ascorbate and a concomitant decrease in reduced ascorbate. ${ }^{47}$ Whilst dietary deficiency of vitamin $\mathrm{C}$ has been shown to increase light-induced ROS damage in guinea pigs, dietary vitamin $\mathrm{C}$ supplementation has been shown to protect against such light-induced damage in rats. ${ }^{29,47}$ In a study comparing two closely related species of spiny mice, the concentration of vitamin C in the aqueous humour of the diurnal species was found to be 35 times higher than that of the nocturnal species. It was suggested that this represented an adaptive response of the diurnal mouse in order for it to better withstand the free radicals generated by solar radiation. ${ }^{48}$

Nutrition may influence lipofuscin accumulation in several ways. In addition to the effects of dietary content of vitamin A, C and E discussed above, reduction in either total food intake or in caloric intake has been shown to slow lipofuscin accumulation in rats. ${ }^{49}$

\section{ROLE OF RPE CELL LYSOSOMAL PROTEASES}

Incomplete proteolytic digestion of ROS within the lysosomes of RPE cells may also play an important role in lipofuscin accumulation. Indeed, it has been proposed that the protein components of ROS are the major precursors of RPE lipofuscin. ${ }^{4,34,50}$ Intravitreal injection of the specific cysteine protease inhibitor leupeptin has been shown to impair the digestion of phagocytosed ROS and result in rapid accumulation of lipofuscin-like material within the RPE cells. ${ }^{34,50}$ In vitro experiments have reproduced these findings and confirmed that specific protease inhibitors can impair ROS degradation by RPE cells without diminishing the rate of ROS phagocytosis. ${ }^{25,51}$ It has been hypothesised that either primary failure of the lysosomal proteases (as might occur with increasing age) or inhibition of the proteases by other factors (namely oxidatively damaged ROS components) could allow non-oxidative amino acid modifications to occur and hence lipofuscin to accumulate. ${ }^{4,34}$ The effect that antioxidant deficiency has on promoting lipofuscin formation may then be viewed as an indirect result of RPE protease inhibition by the oxidised ROS and not a result of the direct transformation of oxidised ROS components into lipofuscin. ${ }^{4}$ This would also explain why lipofuscin cannot be produced in vitro by oxidising tissues. ${ }^{9,39}$

\section{EFFECTS OF AGE}

The progressive increase in cellular lipofuscin is one of the most characteristic features of ageing seen in RPE cells. ${ }^{7}$ The area of each macular RPE cell profile occupied by lipofuscin has been found to increase from $1 \%$ in first decade of life to as much as $19 \%$ in the 81 - to 90 -year-old age group. ${ }^{1}$ Somewhat surprisingly, the fastest rate of lipofuscin accumulation occurs in the first two decades of life. ${ }^{1,2,7}$ With increasing age there is an increase in the lipid content of the lipofuscin granules and a reduction in the amount of polyunsaturated fatty acyl chains. ${ }^{12,52}$ Histopathological studies have shown that fusion of primary lysosomes with lipofuscin granules is 
common in older eyes, suggesting that repeated unsuccessful attempts at digesting the material are occurring. ${ }^{52}$ As illustrated in Fig. 1, there is also a higher rate of lipofuscin granule fusion with melanosomes to form complex granules with advancing age. ${ }^{1,5}$

For reasons detailed above, there has been increasing interest in the possible influence of agerelated changes in RPE enzyme activity. The main lysosomal protease of RPE cells responsible for ROS digestion is thought to be cathepsin D. ${ }^{1}$ Ageing rat organs have been shown to accumulate immunologically active, but enzymatically inactive, cathepsin D. ${ }^{53}$ In vitro experiments on cultured human RPE cells have revealed that there is a decline in extracellular cathepsin D release from the basal surface of the cells with increasing donor age. ${ }^{54} \mathrm{~A}$ separate study on cultured human RPE cells has shown a reduction in the activity of another lysosomal enzyme, $\alpha$-mannosidase, with increasing donor age. Whilst age-dependent reductions in lysosomal enzyme activity could possibly increase the rate of lipofuscin accumulation, an age-dependent decline in retinal antioxidant enzyme activity could also theoretically promote lipofuscin accumulation. Indeed, a study on post-mortem ocular tissue has reported that RPE cell catalase activity declines significantly with advancing age. ${ }^{43}$

\section{RELATION TO RETINAL DISEASE}

The issue of whether lipofuscin accumulation has significant deleterious effects on RPE cell and, consequently, overall retinal function continues to be of great interest. Several mechanisms have been suggested by which lipofuscin may disrupt normal RPE cellular function. Lipofuscin may be a basically inert substance which leads to cellular dysfunction only by means of engorging the cells with nonfunctional waste, causing a reduction in functional cytoplasmic space and distortion of cellular architecture which result in progressive failure of metabolic processes. ${ }^{29,55,56}$ On the other hand, lipofuscin granules may slowly leach abnormal molecular fragments which have some toxicity to normal cellular function. ${ }^{56}$ The only currently identified fluorophore of RPE lipofuscin ( $N$-retinylidene- $N$ retinylethanolamine) is both a detegent and a weak base and when present in sufficient quantities could be expected to inactivate lysosomal enzymes and eventually cause lysis of the lysosomal membrane and cell death. ${ }^{13}$ Some authors have also implicated the autofluorescent properties of lipofuscin as a possible contributing factor. It has been suggested that following absorption of shorter-wavelength light by lipofuscin, radiation re-emitted at other wavelengths inside the RPE cells could lead to further photoçhemical damage. ${ }^{29}$
Histopathological case studies probably provide the strongest available evidence linking lipofuscin to RPE cell dysfunction, although they are unable to prove a direct causal relationship. An abnormal accumulation of lipofuscin in the RPE cells has been noted to be associated with degeneration of RPE cells and adjacent photoreceptors in a dominantly inherited pigment epithelial dystrophy affecting dogs and in chronic lead subacetate poisoning in rabbits. ${ }^{57}$ Lipofuscin accumulation in RPE cells has also been correlated with photoreceptor death in histopathological studies on humans. Photoreceptor loss in Caucasians has been reported to be very significantly and directly correlated with the lipofuscin concentration of the apposing RPE cells. ${ }^{26}$

A particularly controversial topic is the association between lipofuscin and age-related macular degeneration, the commonest cause of blindness in Western society and a disease considered by many to be principally caused by gradual RPE cell failure. ${ }^{58-62}$ As suggested by its title, the strongest risk factor identified for development of age-related macular degeneration is chronological age. ${ }^{47,63}$ As noted above, RPE cell lipofuscin content is also highly correlated with age. ${ }^{1,7}$ One histopathological study noted that the ratio of photoreceptors to RPE cells was higher in the macula than in the paramacula or equatorial region and that the number of photoreceptors per RPE cell profile increased with age. The age-related increase in the ROS burden on individual RPE cells was found to be particularly pronounced at the macula, the favoured anatomical site of disease in age-related macular degeneration. ${ }^{26}$ In the 'dry', atrophic form of age-related macular degeneration which culminates in geographic atrophy, the RPE cells show massive lipofuscin accumulation, loss of cellular organelles, nuclear displacement, and loss of apical microvilli prior to cell death and loss. ${ }^{62}$

Drusen are extracellular deposits that form beneath the RPE cells in age-related macular degeneration. Although lipofuscin and drusen are both considered to be forms of cellular debris produced by the RPE cells, they have distinctly different histological features and their precise relationship is not established. ${ }^{61,64}$ However, the RPE cell atrophy seen in both primary age-related RPE atrophy and drusen-related atrophy precisely parallels the topographic distribution of lipofuscin across the fundus, increasing toward the posterior pole, but tending relatively to spare the centre of the fovea ${ }^{62}$ The process of RPE cell membrane blebbing and cytoplasm extrusion into Bruch's membrane may contribute to drusen formation. ${ }^{56,65,66}$ This finding has been described in age-related macular degeneration $^{66}$ and in aged human eyes ${ }^{67}$ and is an expected finding in cells which contain excessive amounts of 
lysosomotropic detergent such as the recently identified lipofuscin fluorophore $N$-retinylidene- $N$ retinylethanolamine. ${ }^{13}$

Fundus flavimaculatus (Stargardt's disease) and vitelliform macular degeneration (Best's macular dystrophy) are two RPE diseases of unknown aetiology associated with abnormal accumulation of lipofuscin in the RPE cells. Fundus flavimaculatus is the collective name given to a group of inherited macular dystrophies, the majority of which are transmitted in autosomal recessive fashion. ${ }^{68}$ In fundus flavimaculatus the RPE cells contain up to 7 times more lipofuscin than normal and this is associated with RPE cell degeneration and secondary atrophy of photoreceptors and choriocapillaris. ${ }^{68,69}$ In vitelliform macular degeneration, a disease with autosomal dominant inheritance, there is a marked accumulation of lipofuscin both within and beneath the RPE cells. Even at an early stage in the disease, the electro-oculogram is subnormal due to loss of the ability of the RPE cells to maintain a normal transepithelial potential. Later there is progression to frank dysfunction and degeneration of the macula ${ }^{70}$ Finally, several conditions which secondarily cause RPE dysfunction are also associated with excessive lipofuscin accumulation in the RPE cells. Such examples include the retinal pigment epitheliopathy associated with choroidal melanoma $^{70,71}$ and severe ocular trauma. ${ }^{72}$

Although there is an association between RPE lipofuscin accumulation and disease of the retinal pigment epithelium, firm evidence that lipofuscin is directly responsible for RPE cell dysfunction is still awaited. Despite the attraction of the hypotheses which implicate lipofuscin in the pathogenesis of RPE disease, it is still possible that excessive accumulation of lipofuscin is simply an expression of preceding RPE cell dysfunction.

Key words: Ageing, Age-related macular degeneration, Antioxidants, Lipofuscin, Retinal pigment epithelium (RPE), Stargardt's disease.

\section{REFERENCES}

1. Feeney-Burns L, Hilderbrand ES, Eldridge S. Aging human RPE: morphometric analysis of macular, equatorial and peripheral cells. Invest Ophthalmol Vis Sci 1984;25:195-200.

2. Feeney-Burns L, Eldred GE. The fate of the phagosome: conversion to 'age pigment' and impact in human retinal pigment epithelium. Trans Ophthalmol Soc UK 1983;103:416-21.

3. Boulton M, McKechnie NM, Breda J, Bayly M, Marshall J. The formation of autofluorescent granules in cultured human RPE. Invest Ophthalmol Vis Sci 1989;30:82-9.

4. Katz ML. Incomplete proteolysis may contribute to lipofuscin accumulation in the retinal pigment epithelium. Adv Exp Med Biol 1989;266:109-16.

5. Feeney-Burns L, Berman ER, Rothman H. Lipofuscin of the human retinal pigment epithelium. Am J Ophthalmol 1980;90:783-91.

6. Wing GL, Blanchard GC, Weiter JJ. The topography and age relationship of lipofuscin concentration in the retinal pigment epithelium. Invest Ophthalmol Vis Sci 1978;17:601-7.

7. Weiter JJ, Delori FC, Wing GL, Fitch KA. Retinal pigment epithelial lipofuscin and melanin and choroidal melanin in human eyes. Invest Ophthalmol Vis Sci 1986;27:145-52.

8. Eldred GE, Katz ML. Fluorophores of the human retinal pigment epithelium: separation and spectral characterisation. Exp Eye Res 1988;47:71-86.

9. Eldred GE, Katz ML. The lipid peroxidation theory of lipofuscinogenesis cannot yet be confirmed. Free Radic Biol Med 1991;10:445-7.

10. Eldred GE, Miller GE, Stark WS, Feeney-Burns L. Lipofuscin: resolution of discrepant fluorescence data. Science 1982;216:757-9.

11. Eldred GE. Vitamins $A$ and $E$ in RPE lipofuscin formation and implications for age-related macular degeneration. Prog Clin Biol Res 1989;314:113-29.

12. Bazan HE, Bazan NG, Feeney-Burns L, Berman ER. Lipids in human lipofuscin-enriched subcellular fractions of two age populations: comparison with rod outer segments and neural retina. Invest Ophthalmol Vis Sci 1990;31:1433-43.

13. Eldred GE, Lansky MR. Retinal age pigments generated by self-assembling lysosomotropic detergents. Nature 1993;361:724-6.

14. Katz ML, Drea CM, Eldred GE, Hess HH, Robinson WG Jr. Influence of early photoreceptor degeneration on lipofuscin in the retinal pigment epithelium. Exp Eye Res 1986;43:561-73.

15. Robinson WG Jr, Kuwabara T, Bieri JG. Deficiencies of vitamins $\mathrm{E}$ and $\mathrm{A}$ in the rat: retinal damage and lipofuscin accumulation. Invest Ophthalmol Vis Sci 1980;19:1030-7.

16. Katz ML, Norberg M, Stientjes HJ. Reduced phagosomal content of the retinal pigment epithelium in response to retinoid deprivation. Invest Ophthalmol Vis Sci 1992;33:2612-8.

17. Young RW. The renewal of the photoreceptor cell outer segments. J Cell Biol 1967;33:61-72.

18. Newsome DA. Retinal pigmented epithelial cell culture: current applications. Trans Ophthalmol Soc UK 1983;103:458-66.

19. Feeney L, Mixon RN. An in vitro model of phagocytosis in bovine and human retinal pigment epithelium. Exp Eye Res 1976;22:533-48.

20. Bok D. Retinal photoreceptor-pigment epithelium interactions. Friedenwald Lecture. Invest Ophthalmol Vis Sci 1985;26:1659-94.

21. Hayasaka S, Hara S, Mizuno K. Degradation of rod outer segment proteins by cathepsin D. J Biochem 1975;78:1365-7.

22. Zimmerman WF, Godchaux W, Belkin M. The relative proportions of lysosomal enzyme activities in bovine retinal pigment epithelium. Exp Eye Res 1983;36: 151-8.

23. Hayasaka S. Lysosomal enzymes in ocular tissues and diseases. Surv Ophthalmol 1983;27:245-58.

24. Wyszynski RE, Bruner WE, Cano DB, Morgan KM, Davis CB, Sternberg P. A donor-age-dependent change in the activity of alpha-mannosidase in human cultured RPE cells. Invest Ophthalmol Vis Sci 1989;30:2341-7.

25. Rakoczy PE, Mann K, Cavaney DM, Robertson T, Papadimitriou J, Constable IJ. Detection and possible 
functions of a cysteine protease involved in digestion of rod outer segments by retinal pigment epithelial cells. Invest Ophthalmol Vis Sci 1994;35:4100-8.

26. Dorey CK, Wu G, Ebenstein D, Garsd A, Weiter JJ. Cell loss in the aging retina: relationship to lipofuscin accumulation and macular degeneration. Invest Ophthalmol Vis Sci 1989;30:1691-9.

27. Feeney-Burns L, Gao CL, Berman ER. The fate of immunoreactive opsin following phagocytosis by pigment epithelium in human and monkey retinas. Invest Ophthalmol Vis Sci 1988;29:708-19.

28. Boulton M, Marshall J. Repigmentation of human retinal pigment epithelial cells in vitro. Exp Eye Res 1985;41:209-18.

29. Young RW. Solar radiation and age-related macular degeneration. Surv Ophthalmol 1988;32:252-69.

30. Feeney L. The phagosomal system of the pigment epithelium: a key to retinal disease. Invest Ophthalmol Vis Sci 1973;12:635-8.

31. Rakoczy P, Kennedy C, Thompson-Wallis, Mann K, Constable I. Changes in retinal pigment epithelial cell autofluorescence and protein expression associated with phagocytosis of rod outer segments. Biol Cell 1992;76:49-54.

32. Katz ML, Eldred GE. Retinal light damage reduces autofluorescent pigment deposition in the retinal pigment epithelium. Invest Ophthalmol Vis Sci 1989;30:37-43.

33. Boulton M, Marshall J. Effects of increasing numbers of phagocytic inclusions on human retinal pigment epithelial cells in culture: a model for aging. $\mathrm{Br} \mathrm{J}$ Ophthalmol 1986;70:808-15.

34. Ivy GO, Kanai S, Ohta M, Smith G, Sato Y, Kobayashi M, Kitani K. Lipofuscin-like substances accumulate rapidly in brain, retina and internal organs with cysteine protease inhibition. Adv Exp Med Biol 1989;266:31-45.

35. Katz ML, Stone WI, Dratz EA. Fluorescent pigment accumulation in the retinal pigment epithelium of antioxidant-deficient rats. Invest Ophthalmol Vis Sci 1978;17:1049-58.

36. Tappel AL. Biological antioxidant protection against lipid peroxidation damage. Am J Clin Nutr 1970;23:1137-9.

37. Chio KS, Reiss U, Fletcher B, Tappel AL. Peroxidation of subcellular organelles: formation of lipofuscinlike fluorescent pigments. Science 1969;166:1535-6.

38. Chio KS, Tappel AL. Synthesis and characterisation of the fluorescent products derived from malonaldehyde and amino acids. Biochemistry 1969;8:2821-7.

39. Eldred GE, Katz ML. The autofluorescent products of lipid peroxidation may not be lipofuscin-like. Free Rad Biol Med 1989;7:157-63.

40. Katz ML, Stientjes HJ, Gao CL, Christianson JS. Ironinduced accumulation of lipofuscin-like fluorescent pigment in the retinal pigment epithelium. Invest Ophthalmol Vis Sci 1993;34:3161-71.

41. Fite KV, Bengston L, Donaghey B. Experimental light damage increases lipofuscin in the retinal pigment epithelium of Japanese quail (Coturnix coturnix japonica). Exp Eye Res 1993;57:449-60.

42. Newsome DA, Dobard EP, Liles MR, Oliver PD. Human retinal pigment epithelial cells contain two distinct species of superoxide dismutase. Invest Ophthalmol Vis Sci 1990;31:2508-13.

43. Liles MR, Newsome DA, Oliver PD. Antioxidant enzymes in the aging human retinal pigment epithelium. Arch Ophthalmol 1991;109:1285-8.

44. Hayes KC. Retinal degeneration in monkeys induced by deficiencies of vitamin $\mathrm{E}$ or $\mathrm{A}$. Invest Ophthalmol Vis Sci 1978;13:499-510.

45. Penn JS, Thum LA, Awner S. Protective effect of vitamin $E$ in oxygen-induced retinopathy in the rat. Invest Ophthalmol Vis Sci 1989;30(Suppl):3090.

46. Katz ML, Robinson WG Jr, Herrmann RK, Groome AB, Bieri JG. Lipofuscin accumulation resulting from senescence and vitamin E deficiency: spectral properties and tissue distribution. Mech Ageing Dev 1984;25:149-59.

47. Tso MO. Pathogenic factors of aging macular degeneration. Ophthalmology 1985;92:628-35.

48. Gerster H. Review: antioxidant protection of the ageing macula. Age Ageing 1991;20:60-9.

49. Katz ML, White HA, Gao CL, Roth GS, Knapka JJ, Ingram DK. Dietary restriction slows age pigment accumulation in the retinal pigment epithelium. Invest Ophthalmol Vis Sci 1993;34:3297-302.

50. Katz ML, Shanker MJ. Development of lipofuscin-like fluorescence in the retinal pigment epithelium in response to protease inhibitor treatment. Mech Ageing Dev 1989;49:23-40.

51. Kennedy CJ, Rakoczy PE, Robertson TA, Padadimitriou JM, Constable IJ. Kinetic studies on phagocytosis and digestion of rod outer segments by human retinal pigment epithelial cells in vitro. Exp Cell Res 1994;210:209-14.

52. Feeney L. Lipofuscin and melanin of human retinal pigment epithelium: fluorescence, enzyme cytochemical, and ultrastructural studies. Invest Ophthalmol Vis Sci 1978; 17:583-600.

53. Wiederanders B, Oelke B. Accumulation of inactive cathepsin D in old rats. Mech Ageing Dev 1984;24:26571.

54. Wilcox DK. Vectorial accumulation of cathepsin D in retinal pigmentary epithelium: effects of age. Invest Ophthalmol Vis Sci 1988;29:1205-12.

55. Mainster MA. Light and macular degeneration: a biophysical and clinical perspective. Eye 1987;1:304-10.

56. Young RW. Pathophysiology of age-related macular degeneration. Surv Ophthalmol 1987;31:291-306.

57. Aguirre GD, Laties A. Pigment epithelial dystrophy in the dog. Exp Eye Res 1976;23:247-56.

58. Farkas TG, Sylvester V, Archer D, Altona M. The histochemistry of drusen. Am J Ophthalmol 1971;71:1206-15.

59. Gass JDM. Drusen and disciform macular detachment and degeneration. Arch Ophthalmol 1973;90:206-17.

60. Green WR, McDonnell PJ, Yeo JH. Pathologic features of senile macular degeneration. Ophthalmology 1985;92:615-27.

61. Sarks SH. Council lecture. Drusen and their relationship to senile macular degeneration. Aust J Ophthalmol 1980;8:117-30.

62. Sarks SH, Sarks J, Killingsworth C. Evolution of geographic atrophy of the retinal pigment epithelium. Eye 1988;2:552-77.

63. Kahn HA, Leibowitz HM, Ganley J, Kini MM, Colton T, Nickerson RJ, Dawber TR. The Framingham Eye Study. I. Outline and major prevalence findings. Am J Epidemiol 1977;106:17-32.

64. Sarks SH. Ageing and degeneration in the macular region: a clinicopathological study. $\mathrm{Br} \mathrm{J}$ Ophthalmol 1976;60:324-41.

65. Ishibashi $\mathrm{T}$, Sorgente $\mathrm{N}$, Patterson R, Ryan SJ. Pathogenesis of drusen in the primate. Invest Ophthalmol Vis Sci 1986;27:184-93.

66. Ishibashi T, Patterson R, Ohnishi Y, Inomata H, Ryan 
SJ. Formation of drusen in the human eye. Am J Ophthalmol 1986;101:342-53.

67. Feeney-Burns L, Gao CL, Tidwell M. Lysosymal enzyme cytochemistry of human RPE, Bruch's membrane and drusen. Invest Ophthalmol Vis Sci 1987;28:1138-47.

68. Birnbach CD, Jarvelainen M, Possin DE, Milam AH. Histopathology and immunohistochemistry of the neurosensory retina in fundus flavimaculatus. Ophthalmology 1994;101:1211-9.

69. Lopez PF, Maumenee IH, de la Cruz Z, Green WR.
Autosomal-dominant fundus flavimaculatus: clinicopathologic correlation. Ophthalmology 1990;97:798809.

70. Weingeist TA, Kobrin JL, Watzke RC. Histopathology of Best's macular dystrophy. Arch Ophthalmol 1982;100:1108-14.

71. Damato BE, Foulds WS. Tumour-associated retinal pigment epitheliopathy. Eye 1990;4:382-7.

72. Ko MK, Lee WR, McKechnie NM, Hall-Parker B. Post-traumatic hyperlipofuscinosis in the human retinal pigment epithelium. Br J Ophthalmol 1991;75:54-60. 